\title{
Predicción de fenómenos arrítmicos a través de la variabilidad de la frecuencia cardíaca en caninos
}

\author{
Buzzano, O. ${ }^{1}$; Mercado, M. ${ }^{2}$; Lightowler, C. ; Pérez Valega, E. ${ }^{3}$; Maidana, H. ${ }^{3}$ \\ ${ }^{1}$ Unidad de Cardiología, ${ }^{2}$ Unidad de Fisioterapia, Hospital Escuela, Facultad de Ciencias Veterinarias, UBA, \\ Chorroarín 280, Buenos Aires (1427), E-mail: obuzzano@fvet.uba.ar. ${ }^{3}$ Cátedra de Semiología, Facultad \\ de Ciencias Veterinarias, UNNE, Sargento Cabral 2139, Corrientes (3400), Argentina.
}

\begin{abstract}
Resumen
Buzzano, O.; Mercado, M.; Lightowler, C.; Pérez Valega, E.; Maidana, H.: Predicción de fenómenos arrítmicos a través de la variabilidad de la frecuencia cardíaca en caninos. Rev. vet. 23: 1, 55-58, 2012. El estudio de la variabilidad de la frecuencia cardíaca (VFC) es una de las técnicas que permiten conocer el balance y efecto de ambas divisiones del sistema nervioso autónomo sobre el corazón del ser humano. El predominio simpático predispone a las arritmias cardíacas. El estudio consiste en realizar una exploración electrocardiográfica ambulatoria (Holter) y compilar las variaciones que se producen en el intervalo RR entre cada latido cardíaco. De acuerdo a los datos obtenidos tras el correspondiente procesamiento estadístico se desprende que en el perro portador de enfermedades cardíacas la VFC disminuye tal como acontece en el ser humano y, por lo tanto, cumpliría la misma función como predictor de sucesos arrítmicos.
\end{abstract}

Palabras clave: perro, variabilidad frecuencia cardíaca, Holter, arritmias cardíacas.

\begin{abstract}
Buzzano, O.; Mercado, M.; Lightowler, C.; Pérez Valega, E.; Maidana, H.: Heart rate variability in dogs. Its usefulness as a predictor of arrhythmic events. Rev. vet. 23: 1, 55-58, 2012. The study of heart rate variability (HRV) allows in humans to know the balance and effect of both divisions of the autonomic nervous system of the heart. The sympathetic predominance predisposes to cardiac arrhythmias. This study was performed through an ambulatory ECG (Holter), with statistical analysis of the variations that occur in the RR interval between each heartbeat. According to the data obtained and the corresponding statistical analysis it can be concluded that, for the diseases of the canine heart, the HRV decreases similarly to humans, thus having the same usefulness as a predictor of arrhythmic events.
\end{abstract}

Key words: dog, heart rate variability, Holter, cardiac arrhythmias.

\section{INTRODUCCIÓN}

La variabilidad de la frecuencia cardiaca (VFC) es definida como el ritmo biológico bifásico gobernado por el sistema nervioso autónomo (SNA) e implementado a través del automatismo del nódulo sinusal ${ }^{8}$ . Este fenómeno es controlado y modulado por acción central y/o periférica, originando períodos circadianos fisiológicos en la $\mathrm{VFC}^{9}$. La relevancia del análisis acerca del comportamiento de las variaciones duales de la frecuencia cardíaca en el canino es que permite conocer cuándo predomina el tono autonómico simpático o parasimpático, convirtiendo a este fenómeno en un importante marcador de la posibilidad de padecer $\operatorname{arritmias}^{4,5}$.

Recibido: 4 abril 2012 / Aceptado: 14 mayo 2012

Proyecto VE006 (Secretaría de Ciencia y Técnica UBA)
La hipótesis de trabajo planteó demostrar que bajo diferentes estímulos vegetativos, el sistema cardiocirculatorio presenta disminución de la VFC como consecuencia del predo-minio del tono simpático, particularmente en pacientes que presentan distintas entidades cardíacas patológicas. Para la comprobación de la hipótesis se utilizó la técnica de estudio de la VFC en el denominado "dominio de tiempo". El procesamiento informático analiza la señal eléctrica grabada en un estudio Holter y expresa, en milisegundos, los valores de los sucesivos intervalos RR.

El procedimiento básico establece la necesidad de trabajar solamente sobre complejos sinusales normales pares e impares (descartando especialmente los prematuros), expresando la varianza interlatido. De este estudio surgen distintas variables, siendo las más representativas para el análisis de la VFC las siguientes: el desvío estándar (SDNN), el promedio de todos los 
desvíos estándares, la raíz cuadrada de la media de los cuadrados de las diferencias entre intervalos contiguos, el índice de los desvíos estándares del espectro de la relación baja/ alta frecuencia espectral y los cálculos de entropía aproximada (asumiendo que el modelo es lineal con patrón repetitivo de señal) ${ }^{7}$.

\section{MATERIAL Y MÉTODOS}

Para el presente estudio se incluyeron 40 caninos, 20 libres de enfermedades y 20 con distintas alteraciones cardíacas. Para el registro ambulatorio se utilizó un equipo Holter digital marca NortEast Monitoring con grabadora de tres canales de registro simultáneo, cuyo tamaño aproximado es el de un paquete de cigarrillos. Todo el procesamiento de la misma se realiza a través de los botones de color gris (Figura 1).

Se emplearon electrodos autoadhesivos pediátricos colocados en una posición preestablecida y la metodología de preparación, conexión y colocación del equipo fue la misma para todos los caninos estudiados. Los electrodos fueron sostenidos por una faja elástica sobre la cual se montó un arnés con un bolsillo que alojó a la grabadora (Figura 2). El período de análisis fue de seis horas continuas de grabación.

Los datos resultantes se analizaron con el programa Statistix 8.0 con un nivel de significación del 5\%. Se determinaron los valores de la estadística descriptiva, luego se comprobó la normalidad de las muestras por medio de la prueba de Shapiro-Wilks modificada y se realizó un análisis de varianza.

\section{RESULTADOS}

Los resultados se muestran en Tablas 1 y 2 y Figuras 3 y 4. Los valores asentados en Tabla 1 (expresados en milisegundos) corresponden a los guarismos calculados por el software del equipo e indican la variación de tiempo interlatido, separados para los pacientes normales y enfermos.

La tabla 2 agrupa los resultados de la estadística descriptiva, para caninos normales y patológicos, cuyos datos de origen corresponden a los valores asentados en la tabla 1 .

En la Figura 3 se demuestra la mayor variabilidad de la frecuencia cardiaca en los caninos normales y menor en los patológicos.

En la Figura 4 (Esquema de Poincaré) se muestra la diferencia de distribución de los intervalos RR cuando se mide la variabilidad de la frecuencia cardíaca en dominio de tiempo. A la izquierda se muestra un paciente normal donde se observa una amplia distribución de intervalos RR lo que indica un buen balance neurovegetativo. A la derecha se presenta el esquema de un paciente con una cardiomiopatía dilatada donde se demuestra la escasa distribución de los intervalos $\mathrm{RR}$ indicando un predominio de la división simpática y una mayor predisposición a las arritmias cardíacas. Los cambios analizados para la VFC permiten com-
Tabla 1. Valores de VFC obtenidos.

\begin{tabular}{ll}
\hline normales & $138 ; 111 ; 164 ; 133 ; 118 ; 151 ; 131 ; 105 ; 124 ;$ \\
& $141 ; 125 ; 115 ; 136 ; 146 ; 157 ; 109 ; 154 ; 143 ;$ \\
& $160 ; 149$ \\
\hline patológicos & $125 ; 103 ; 142 ; 120 ; 108 ; 133 ; 116 ; 93 ; 117 ;$ \\
& $129 ; 101 ; 99 ; 123 ; 139 ; 140 ; 103 ; 147 ; 126 ;$ \\
& $145 ; 136$ \\
\hline
\end{tabular}

Tabla 2. Valores de la estadística descriptiva.

\begin{tabular}{lcc}
\hline & normales & patológicos \\
\hline $\mathrm{n}$ & 20 & 20 \\
media & 135,500 & 122,25 \\
desvío estándar & 17,875 & 16,783 \\
varianza & 390,530 & 281,67 \\
valor mínimo & 105,000 & 93,000 \\
mediana & 137,000 & 124,000 \\
valor máximo & 164,000 & 147,000 \\
\hline
\end{tabular}

probar la existencia de diferencias significativas en las variables bajo estudio.

Los datos emergentes de la estadística descriptiva para la variable principal estudiada SDNN, así como la comprobación de normalidad con el test de ShapiroWilks y la comprobación de independencia a través de la prueba " $t$ " para dos muestras, seguida del análisis de varianza, indican la existencia de diferencias estadísticamente significativas entre los caninos normales

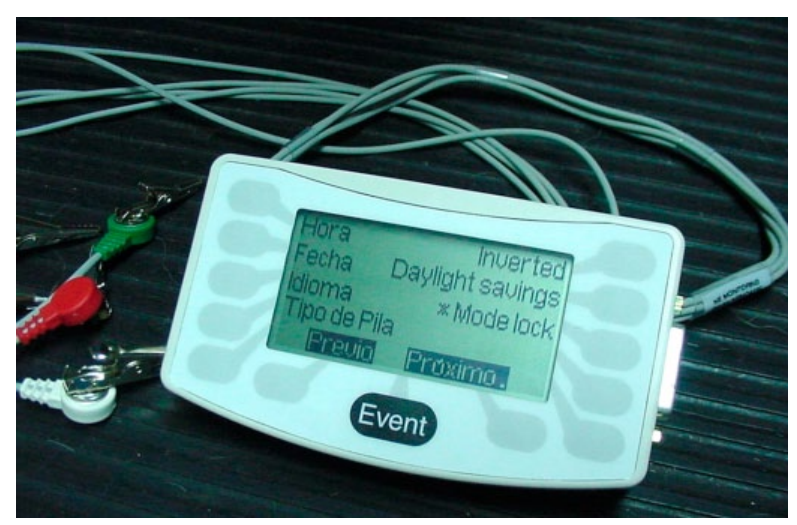

Figura 1. Grabadora del equipo utilizado.

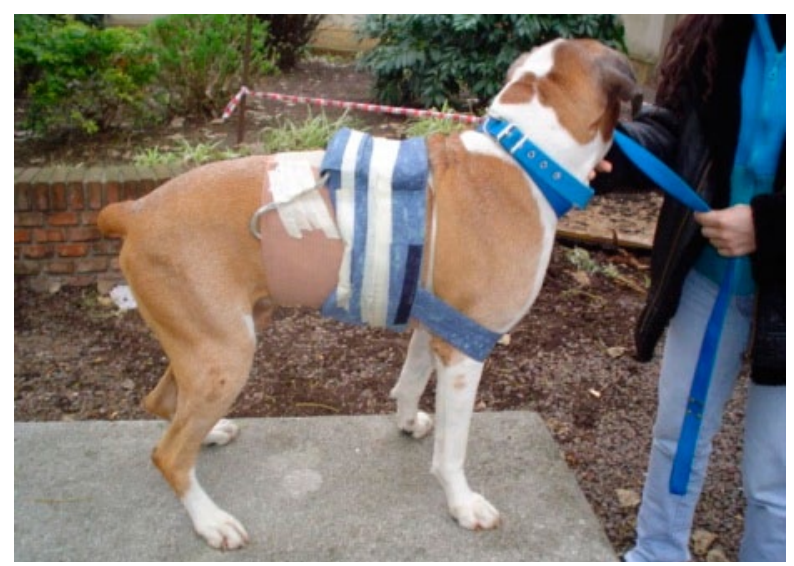

Figura 2. Sujeción del grabador Holter sobre el paciente. 
y patológicos estudiados, como puede apreciarse en el siguiente resumen tabulado:

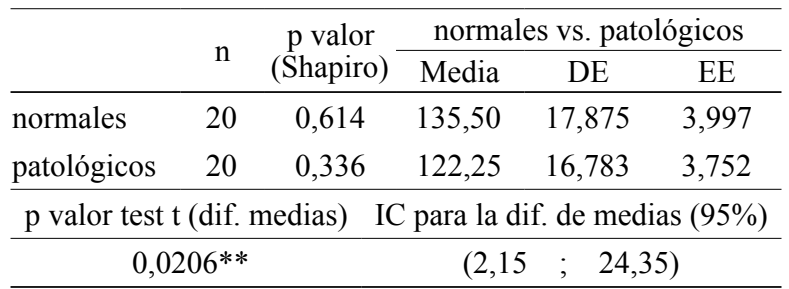

Las medias de ambas poblaciones fueron significativamente diferentes a un nivel del 5\%.

\section{DISCUSIÓN}

En la investigación de la VFC en caninos se debe tener en cuenta el efecto que produce la variación circadiana natural sobre la frecuencia cardíaca, emplear un solo método estandarizado de análisis, así como respetar el tiempo de registro del estudio ambulatorio (Holter) con el objeto de evitar la producción de sesgo estadístico. En paralelismo con las investigaciones realizadas en el ser humano, es dable esperar que la especie canina presente igualmente una disminución de la VFC, tanto con el avance de la edad por normal degeneración del sistema neurovegetativo, así como por la presencia de diversas patologías cardíacas.

Desde el punto de vista técnico, la buena resolución en la captura de la señal eléctrica inicial para que la conversión analógica-digital sea correcta, la capacidad de filtrar el componente señal-ruido y el reconocimien- to de los complejos QRSs normales son puntos críticos a superar para que el análisis resultante represente la señal de ingreso o electrocardiograma original y los resultados finales puedan ser expresados en el dominio de tiempo.

La información obtenida puede ser aplicada para la estratificación del riesgo arrit-mogénico en la insuficiencia cardíaca, en las miocardiopatías (dilatada, restrictiva, arritmo-génica), en la isquemia miocárdica, en la obstrucción del tracto de salida del ventrículo izquierdo en los casos de estenosis subaórtica congénita o en la cardiomiopatía hipertrófica y otros defectos congénitos con idéntico componente obstructivo.

Fluctuaciones muy amplias de la VFC (taquicardiabradicardia) pueden causar inestabilidad eléctrica e iniciar o mantener eventos arrítmicos. La estimulación catecolaminérgica incrementa significativamente el riesgo de muerte por arritmias severas, sobre todo en razas predispuestas (boxer, doberman, pastor alemán) ${ }^{1-3,6}$, al disminuir el umbral eléctrico, factor que aumenta la probabilidad de inducción de una arritmia ventricular.

Sobre la base de los datos obtenidos y su posterior procesamiento estadístico puede concluirse que es factible inferir que en el perro, al igual que lo demostrado ya en seres humanos, la disminución de los valores resultantes del análisis de la VFC, serían predictores importantes de la aparición de arritmias severas.

El método utilizado permitió valorar adecuadamente la VFC y confirmar la hipótesis planteada, demostrando así el efecto de la modulación parasimpática sobre la frecuencia cardiaca. De acuerdo a la experien-
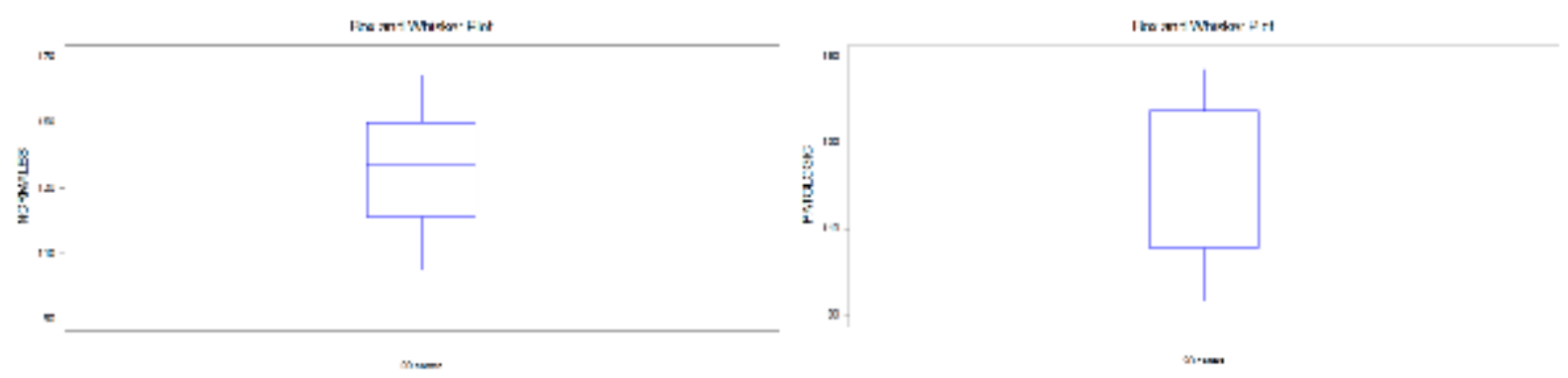

Figura 3. Esquema de caja y bigote para la distribución de los valores procesados en perros normales (izquierda) y patológicos (derecha).
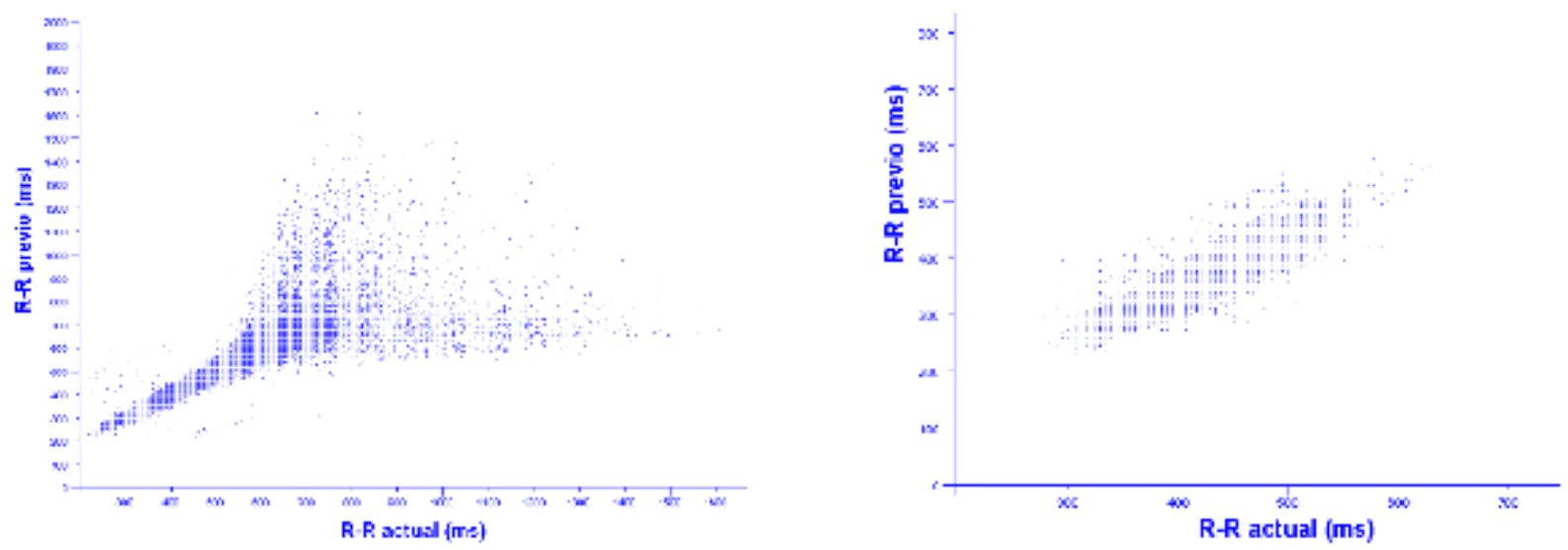

Figura 4. Distribución de los intervalos RR en un esquema de Poincaré en un canino sano (izquierda) y otro con cardiomiopatía dilatada (derecha). 
cia adquirida durante el presente estudio, el aspecto más importante para evitar la alteración de los resultados finales del procesamiento informático es respetar escrupulosamente la igualdad del tiempo de registro Holter en todos los pacientes.

\section{REFERENCIAS}

1. Bonagura JD, Darke PG. 1995. Congenital heart disease. In: Textbook of veterinary internal medicine (Ettinger SF, Feldman EC Ed. ), $2^{\text {nd }}$ ed., Saunders, Philadelphia, p.892.

2. Calvert CA, Hall G, Jacobs GJ. 1997. Clinical and pathologic findings in doberman pinscher with ocult cardiomyopathy dying suddenly or that developed congestive heart failure. JAVMA 210: 505-511.

3. Calvert CA, Jacobs GJ, Pickus CW. 1998. Signalmen survival and prognostic factors in dobermans pinschers with end-stage cardiomyopathy. J Vet Intern Med 11: 323326.
4. Landsberg L, Young J. 1994. Fisiología y farmacología del sistema nervioso autónomo. In: Principios de medicina interna de Harrison (Isselbacher $\mathrm{K}$, Braunwald E, Wilson JD, Martin J, Fauci A, Kasper D Ed.), 13ª ed., Mc Graw-Hill, vol. 1, p. 481-497.

5. Larson SK, Porges SW. 1982. The ontogeny of heart period patterning in the rat. Dev Psychobiol 15: 519-528.

6. Moisen S, Gilmour F, Meyers N. 1990. Inherited ventricular ectopy and sudden death in young german shepherd dogs. J Am Coll Cardiol 15: 152A.

7. Pincus SM, Gladstone IM, Ehrenkranz RA. 1991. A regularity statistic for medical data analysis. J Clin Monit 7: 335-345.

8. Sleight P, La Rovere M, Mortara A. 1955. Physiology and pathophysiology of the heart and blood pressure variability in humans: is power spectral analysis largely an index of baroreflex. Clin Sci (Colch) 88: 103-109.

9. Štefíková H, Šovčíková E, Broniš M. 1986. The circadian rhythm of selected parameters of heart rate variability. Physiol Bohemoslov 35: 227-232. 